\title{
Railway disruption recovery: lessons from airlines
}

\author{
I. Evans
}

Constraint Technologies International, Australia

\begin{abstract}
CTI is combining its products for situation awareness and disruption recovery in airlines with its railway timetabling software to develop a tool for situation awareness and disruption recovery in a railway environment.

Analysis has shown that many concepts are similar, but that the extra complications caused by interactions between train paths require extra visualisation options such as train diagrams and spatial network displays, along with extra constraint checking to identify and prevent conflicts.

It has been found that these extra requirements can fit within the same overall framework as is used for airlines. The extra visualisation options for rail were then seen to in turn provide value for airlines, as the format used for train diagrams is useful to visualise crew connections, and spatial network displays are useful to visualise air corridors. The extra constraint checking required for rail can also be useful for airlines to model flow restrictions placed on congested runways and air corridors.
\end{abstract}

Keywords: disruption management, situational awareness, recovery optimization, airlines, railways, fleet, crew, passengers.

\section{Introduction}

The disruption management process in airlines has been the subject of a large amount of academic study summarised by Kohl et al. [1] and Clausen et al. [2] and is expedited by commercial products offered by several vendors $[3,4]$ that provide integrated fleet disruption management taking into account scheduled maintenance, crew, passengers and cargo. The situation for other modes of transport such as heavy and light rail and buses is much less mature, yet there are many similarities in the problems such that much can be learned and borrowed 
from the airline work. The similarities are most pronounced for scheduled services rather than on-demand services, so that this paper will confine discussion to these.

\section{Terminology}

The disruption management procedure for airlines has been described using a range of terminology, and thus it is necessary to define the terms used in this paper. Here we use the following terms for the three main components:

- Situational Awareness, where controllers of an airline's operations are provided with accurate and timely information about all aspects affecting the operation of a schedule.

- Recovery Scenario Evaluation, where a controller can evaluate the effect of actions that might be taken to recover from disruptions without committing to them.

- Publishing of Schedule Updates, which publishes changes in the schedule to recover from the disruption.

We will also define the term Recovery Optimisation to describe a tool for use during Recovery Scenario Evaluation that automatically produces on request one or more suggestions for recovery scenarios that will minimise the amount of disruption. Much of the academic literature for both airlines and railways concentrates on such tools that provide a single optimal solution, however our experience with airlines has shown that it is not possible to provide a single solution that will be acceptable in practice for all disruptions. There are two reasons for this:

- It is difficult to state a single objective function to be minimised that correctly encapsulates the tradeoffs that are required in all disruption scenarios.

- There is often extra information that is not available electronically when the optimisation is run. An example from airlines is that the time taken for a mechanical fault to be rectified can only be estimated, so that the ability to produce a range of provisional solutions for comparison that are based on different estimates is a very useful part of the decision making process.

Each of these components will be discussed separately in the following sections.

\section{Operator versus network disruption management}

One confusing aspect of the usage of the term disruption management in the transport context is that disruption management occurs at two different levels.

With airlines, disruption management is performed by each airline for its own fleet, and there is a separate level of disruption management that occurs at the level of Air Traffic Control which is concerned with airspace and airport resource usage by all airlines. In this paper we will use the term operator disruption management to refer to the first of these, and network disruption management to refer to the second. 
A similar situation occurs for rail where above-rail operators (also known as train operators) are concerned with disruptions to the operations of their fleet, while below-rail operators (also known as infrastructure managers) are concerned with managing the provision of paths in the event of disruptions to infrastructure availability or the schedules of the above-rail operators. The distinction between these two types of disruption management in rail is less clear where an operator is both the above- and below-rail operator, such as commonly occurs in dedicated metro systems.

The two levels of disruption management are interlinked, but there are well defined systems that allow information to be exchanged between the systems to enable good decisions to be made in the two separate systems. In the airline context, the Air Traffic Control system allocates movement slots to limit movements, and an airline can then make adjustments to its schedule to make best use of its available movement slots. A similar situation occurs with paths requested by an above-rail operator and allocated by a below-rail operator.

In this paper we are suggesting that it is best to keep these two levels of disruption management separate, even for cases where an operator is responsible for both levels. There are several reasons for this:

- The lower level of detail that needs to be considered at the level of operator disruption management enables options to be seen more clearly without having to concentrate on infrastructure details.

- The timeframes involved in operator disruption management are longer than those involved in network disruption management. The extra time available to make these decisions enables better strategic decisions to be made at the higher level.

- Operator disruption management should be concerned with more detailed checks for non-network resources - for example crews, passenger overcrowding and scheduled maintenance for particular vehicles are not considered in the context of network disruption management.

- The systems that control the below-rail infrastructure are complex, and since development and maintenance of these systems is costly and timeconsuming it is sensible to only include essential functionality at this level.

- Separate software used to assist with disruption management currently exists for each level, so it is much easier to take advantage of existing functionality if this separation is maintained.

When seen from this point of view, it can then be seen that much commercial software currently exists for both levels of disruption management for air transport [3-6], but most commercial software for railways concentrates on network disruption management or only handles a limited number of resources in the context of operator disruption management $[7,8]$. It will be further seen that there are many similarities between operator disruption management for airlines and other modes, and this paper will concentrate on showing the possibilities for the use of this technology by above rail operators. 


\section{Situational awareness}

Situational awareness is the most critical factor in the process of disruption management. Tools that make it easy for a controller to quickly comprehend the essential features of a disruption increase both the speed and quality of the recovery process.

A vital component of situational awareness software is the ability to show events in real-time. This requires an event-driven architecture that processes messages as they arrive and immediately updates all visualisations. Such architectures have been in use in an airline context at CTI for 20 years, and we are now extending this technology for use with rail situational awareness.

To understand the full picture of the current situation it is necessary to have the following information:

- The current position and availability of all vehicles.

- Current information about any network restrictions. For airlines, this information includes arrival and departure slot availability at airports. For railways this can include information about available paths including time restrictions caused by factors such as timed track possessions, and other factors such as temporary speed restrictions.

- Forecast information to predict whether any current deviations from the nominal schedule are likely to naturally correct from the built-in recovery times in the schedule, or whether recovery actions need to be taken to prevent problem escalation.

- Information about planned crew duties, rest breaks, connections and location of standby crew. It is important to also be able to check maximum duty and minimum rest break rules to determine if delays will make the currently planned duties illegal. Crew information is particularly important in longdistance networks.

- Information about passenger numbers and itineraries (and/or cargo for freight operations), or estimates if these are not accurately known. This is vital for determination of the impact of a disruption, and particularly so in a railway context since overcrowding has a significant effect on dwell times, and thus can affect running times significantly.

- Information about scheduled maintenance times and locations for vehicles. This is important if repositioning a vehicle for planned maintenance would be very expensive, as is normally the case for airlines and long-distance rail lines.

A range of visualisation formats have been used for both airline and railway disruption management, and as different formats have different strengths for different classes of disruption the most desirable option is to have all formats available so that the controller can choose the most suitable for any given situation. It is also interesting to note that most of the formats are also applicable to other transport types, although the amount of usage of the different formats is likely to differ between modes. 


\subsection{Gantt chart}

Gantt charts (Figure 1(a)) provide easy visualisation of resource usage over time.

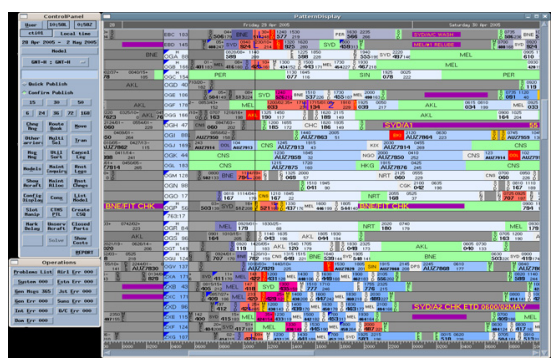

(a) Gantt chart

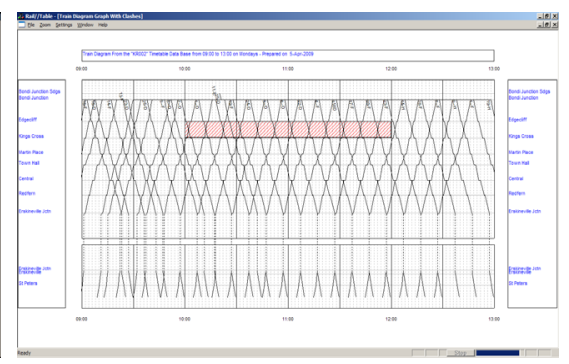

(b) Train diagram

Figure 1: Main visualisation displays for airlines and rail.

For airline operations, it is typical to show the actual or planned legs for each vehicle as the prime visualisation tool. This display is typically annotated to show the origin and destination of each leg as well as the flight number, with colour coding used to show problems such as late departures or arrivals, crew or passenger connection problems, ATC slot problems, insufficient turnaround times at airports, aircraft unavailability and problems meeting scheduled maintenance locations or times.

For rail operations, this can be used in a similar way to show the trips planned for each vehicle while highlighting any problems. In order to avoid excessive clutter, only trip segments between major stations or junctions would be shown at this level, with the stopping pattern information being available by clicking on each trip segment.

A Gantt chart has the disadvantage of not highlighting the interactions between vehicles, which is less of a problem in an airline context but is a significant factor for rail. Thus for rail it is likely that the prime visualisation tool would instead be a Time-Distance Chart.

\subsection{Time-distance chart}

Time-distance charts (Figure 1(b), commonly known for railways as Train Diagrams or sometimes Service Planning Diagrams) are an excellent method of visualising interactions between services and also any network limitations such as track possessions. 
These can be updated in real time to visualise actual delays and estimated arrival times, and can also have overlaid crew connection information to assist with visualisation of likely crew impacts.

This type of visualisation can also be useful in an airline context to visualise connections between services for vehicles and crew in the context of point-topoint networks with an approximately linear topography such as the east coast of Australia.

\subsection{Platform occupancy diagram}

Diagrams showing occupancy of platforms over a given time such as that shown in Figure 2(a) are commonly used in rail planning, and are also very useful on the day of operation. These are also known as Station Activity Diagrams. This type of visualisation is also well suited to showing airport gate usage.

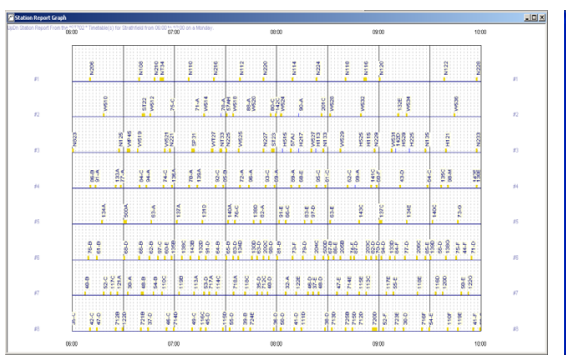

(a) Platform occupancy diagram

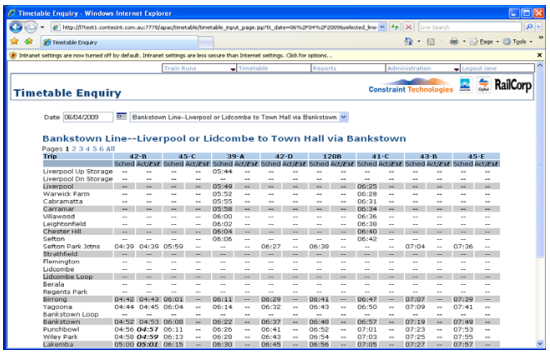

(b) Real time timetable

Figure 2: Other visualisation displays.

One of the critical factors when dealing with metro rail systems in peak periods is the requirement to ensure that the number of passengers arriving at each station can be transported using the available services. In many metro systems, some overcrowding occurs even in the absence of any cancellations. If trains are cancelled, it is vital to ensure that the capacity of subsequent trains on the same line is sufficient to recover from the cancellation, as otherwise the resulting increase in dwell times caused by extreme overcrowding is likely to lead to a decrease in the line capacity and thus an escalating problem. One way of visualising whether this is occurring would be to include estimated passenger figures on the platform occupancy diagram. 


\subsection{Timetable}

A standard means of visualising a railway schedule is the timetable view. Additions showing the actual and estimated times along with any cancellations or changes to stopping patterns as shown in Figure 2(b) are another way of visualising the current situation on the day of operations that is immediately familiar.

\subsection{Spatial displays}

Network displays such as track and signal diagrams shown in Figure 3(a) are used extensively in railways by below-rail operators, but are in general too detailed for higher level planning. Simplified network diagrams may be useful for aboverail operators to visualise alternative routings in complex networks with several alternatives, however. A similar concept may also be useful for airline operators where bad weather can cause flow restrictions through certain areas.

A geospatial display such as that in Figure 3(b) could be useful where it is necessary to take into account options that can use other modes of transport e.g. the use of buses for transport of passengers in the event of a major network disruption.

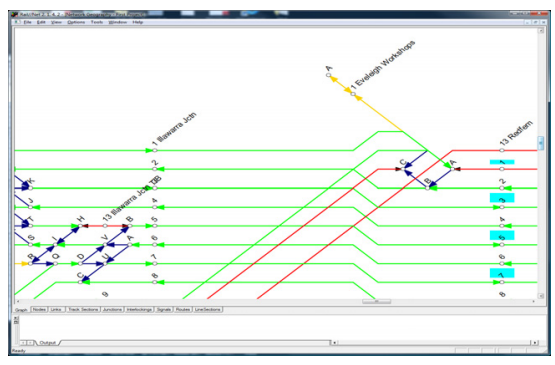

(a) Network diagram

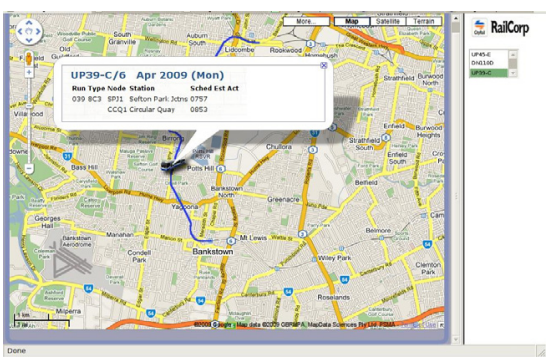

(b) Geospatial display

Figure 3: Spatial visualisation displays.

\subsection{Summary information}

Summary information can be used to evaluate the current situation and to help to decide whether measures need to be taken to aid recovery from a disruption. If a timetable is robust, recovery from small delays is likely to be automatic through the recovery time built in at certain stops. One of the key functions of situational awareness is to understand when the situation is such that this is unlikely to happen and that other measures need to be taken to expedite recovery. 
Table 1: Key performance indicators.

\begin{tabular}{|l|c|c|c|c|c|}
\hline Indicator & $\begin{array}{c}\text { One } \\
\text { week } \\
\text { ago }\end{array}$ & $\begin{array}{c}\text { So far } \\
\text { current } \\
\text { day }\end{array}$ & $\begin{array}{c}\text { Forecast } \\
\text { to } \\
\text { midnight }\end{array}$ & $\begin{array}{c}\text { Total } \\
\text { last } \\
\text { month }\end{array}$ & $\begin{array}{c}\text { Forecast } \\
\text { to end } \\
\text { of month }\end{array}$ \\
\hline Delays > 5 mins & 53 & 30 & 51 & 1510 & 1456 \\
Delays > 10 mins & 32 & 20 & 32 & 923 & 912 \\
Delays > 15 mins & 15 & 10 & 17 & 478 & 456 \\
Delays > 30 mins & 6 & 2 & 5 & 204 & 193 \\
Delays > 60 mins & 3 & 0 & 1 & 56 & 45 \\
Cancellations & 4 & 0 & 2 & 98 & 87 \\
Pax delayed ('1000) & 6.7 & 4.2 & 6.6 & 202 & 195 \\
Delay pax-mins ('1000) & 132 & 79 & 121 & 3962 & 3765 \\
Cancelled maintenance & 1 & 0 & 0 & 11 & 9 \\
Standby crew used & 4 & 2 & 3 & 120 & 105 \\
Crew overtime hours & 25 & 10 & 24 & 753 & 742 \\
\hline
\end{tabular}

Table 2: Remaining problems.

\begin{tabular}{|l|c|}
\hline Type & Count \\
\hline Insufficient turn time 1-5 mins & 5 \\
Insufficient turn time 6-10 mins & 0 \\
Insufficient turn time 11-15 mins & 0 \\
Insufficient turn time $>$ 30 mins & 0 \\
Leg port mismatches & 0 \\
Missed crew connections & 1 \\
Missed scheduled maintenance & 1 \\
\hline
\end{tabular}

In both airlines and railways, periodic on-time performance statistics are published which materially affect the profitability of the operator. For airlines, this effect is often mainly on reputation, although there are financial repercussions if Air Traffic Control slots are consistently missed. For railways, there are often penalty clauses such that operators lose revenue if performance drops below certain values. In such situations, it is useful to be able to track the performance during the current reporting period and to forecast the likely numbers at the end of the period. This information can then be used when choosing recovery options for example to choose to increase the overtime payments to crew in order to avoid excessive service cancellation.

Summary information can then be shown in real time in a manner similar to Table 1, depending on the performance figures important to the operator. 
As it is typically not possible to solve all problems for the rest of the day immediately, it is also important to show the number of remaining problems in a format such as shown in Table 2 .

\section{Recovery scenario evaluation}

Recovery Scenario Evaluation involves a controller making a number of provisional changes in one or more private "what-if?" scenarios to recover from the disruption, and then evaluating the expected outcome from these changes, often by means of comparison of the summary statistics.

Since these changes are being made in a real-time environment, they are stored in a format such that updates to legs that have not been changed in the scenario are reflected as they happen. Updates to legs that have been changed, however, can result in the scenario being updated if it is no longer possible to make the provisional changes. For example, if a scenario plans to swap the lines of flying for two aircraft at port ABC starting from flights 123 and 124, then if either of these flights actually depart from port ABC then the swap is no longer possible and will automatically be removed from the scenario.

The changes that are typically performed in an airline context are:

- Delaying all legs that are to be flown by an aircraft, but reducing the turnaround time between future legs in order to make up time. This is only appropriate for short delays.

- Swapping the lines of flying for one aircraft with another at a port so that an aircraft that has already arrived but has a long scheduled turnaround time can be used to fly the leg that has been planned for an aircraft that is late. This is a good choice when the late aircraft is due to arrive before the scheduled departure of the other aircraft.

- Cancelling a set of legs in a loop (often a return trip). This is appropriate only if the load on the legs is low and the frequency on that route is high, as the passenger disruption would then be limited, or if flow limitations at an airport make it essential.

- Adding or removing a stop for a multi-leg flight. This is not common for airlines, and only makes sense in certain network geographies with several airports are located approximately in a line. The most common use of adding a stop is for situations with strong headwinds where an extra stop is required to take on more fuel.

- Delaying, cancelling or moving scheduled vehicle maintenance activities where this is possible. This can have a cost impact since often a maintenance crew will have been organised at a particular location and time to perform this maintenance.

- Adding another service to cater for stranded passengers.

For an airline, the main criteria against which the impact is assessed against are:

- The on-time performance statistics of the airline, which are published regularly and can be seen as a competitive advantage or disadvantage.

- The total number of affected passengers. 
- The total passenger delay in passenger-minutes.

- The requirement for standby crew or overtime.

- The number of disrupted scheduled vehicle maintenance activities.

Similar actions are possible in the rail context as outlined in Norio et al. [9], but the extra constraints associated with railways would result in a greater emphasis on adding or removing stops, with this being further broken down into the following main options:

- Changing the stopping pattern on a trip section, e.g. from all-stop to express or limited express or vice-versa.

- Terminating a trip at an intermediate station and connecting it to another trip that has been changed to start at that station.

- Changing the departing or meeting orders of trains, e.g. to allow a rapid train to overtake a local train.

For a railway, the main criteria against which the impact is assessed against could be:

- The on-time performance statistics of the railway, which may be tied to penalties if they drop below specified levels.

- The total number of affected passengers.

- The total passenger delay in passenger-minutes.

- The number of trips with severe overcrowding.

- The requirement for standby crew or overtime.

Note that for rail lines where actual passenger numbers are not well known, passenger statistics would have to be estimated based on expected passenger flows. For this reason, these measures are currently mainly used in an informal way, even though they more accurately reflect the perceived passenger service quality, and the extent to which they are used depends on the experience of the traffic controllers.

\section{Publishing of schedule updates}

When an acceptable provisional recovery scenario has been produced, the set of associated changes is published as a single action to ensure that consistency is maintained.

In an airline context, publishing normally involves sending one or more teletype messages to the worldwide SITA or AFS [10] networks, which are then directed to any interested airlines. Situational Awareness tools used by these airlines will then process the received messages to update the current status of the airline's flights or connecting flights that the airline is interested in.

In the rail context, standards for messaging are less mature due to many railways operating on isolated networks.

In Europe, draft messaging standards have been specified in response to Interoperability Directives 96/48/EC (High-Speed), 2001/16/EC (Conventional Rail) and 2004/50 (alignment of High-Speed and Conventional Rail Directives and extension of the scope). Implementation of these standards is progressing across the network. 
In the UK, the SIRI and TransXChange standards have been developed for publishing of real-time public transport information and are in widespread use, although TransXChange is aimed more suited to buses than railways.

\section{Recovery optimisation}

The recovery optimisation problem has been the subject of much study for airlines and for rail.

The problems studied for airlines consider a much greater range of resources, with many considering fleet, scheduled maintenance, airport slots and full passenger itinerary modelling [11], and several also incorporating crew [12].

For railways, most work on recovery optimisation concentrates on the fleet and incorporates quite detailed consideration of the network paths along with some consideration of passenger connections [13], while passenger overcrowding and crew are only considered for simple problems [14] [15].

As crew connection problems can have a large impact on the feasibility of solutions for complex or long-haul networks, and the passenger service quality can have a large impact when comparing the quality of the solution and also the feasibility (due to the effect of overcrowding on dwell times), we consider that extensions of airline recovery optimisation formulations to allow for the more complex constraints of railway paths would be extremely beneficial.

\section{Conclusion}

There are many advantages in considering disruption management in railways at a high level, as this enables consideration of factors such as passenger overcrowding and crew connections. When considered this way, there are many similarities that allow the use of the mature technology used for disruption recovery in airlines to enable more effective disruption recovery for rail.

\section{References}

[1] Kohl, N., Larsen, A., Larsen, J., Rossd, A. \& Tiourine, S., Airline disruption management - perspectives, experiences and outlook. Journal of Air Transport Management, 13(3), pp. 149-162, 2007.

[2] Clausen, J., Larsen, A. \& Larsen, J., Disruption management in the airline industry - concepts, models and methods. University of Denmark, DTU, 2005.

[3] TPAC Operations product. Constraint Technologies International, http://www.constrainttechnologies.com/software/TPAC_Operations/.

[4] iFlight Operations product. IBS Software, http://www.ibsplc.com/fleetscheduling-and-management-software.html.

[5] OSYRIS software suite. Barco, http://www.barco.com/en/ productcategory/29. 
[6] Air Traffic Management products. SELEX Systems Integration, http://www.selex-si-uk.com/markets/air_traffic_management/ atm_products.aspx.

[7] RailEdge Traffic Control \& Dispatch Systems. GE Transport, http://www.getransportation.com/na/en/trafficcontroldispatchsystems.html.

[8] Rail Operations System. Siemens Mobility, http://www.mobility.siemens.com/mobility/en/pub/interurban_mobility/rail _solutions/rail_automation/operations_control_systems.htm.

[9] Norio, T., Yoshiaki, T., Noriyuki, T., Chikara, H. \& Kunimitsu, M., Train rescheduling algorithm which minimizes passengers' dissatisfaction. Lecture Notes in Computer Science, Springer: Berlin and Heidelberg, volume 3533, pp. 829-838, 2005.

[10] AMC and European Fixed Service (AFS). EUROCONTROL, http://www.eurocontrol.int/amc/public/standard_page/aeronautical_fixed_ service.html.

[11] Artigues, C., Bourreau, E., Afsar, H.M., Briant, O. \& Boudia, M., Disruption management for commercial airlines: Methods and results for the ROADEF 2009 challenge. Hyper Articles en Ligne, hal-00492035, 2010.

[12] Petersen, J.D., Solveling, G., Johnson, E.L., Clarke, J.P. \& Shebalov, S., An optimization approach to airline integrated recovery. AGIFORS Airline Operations 2010, 2010.

[13] Tornquist, J., Computer-based decision support for railway traffic scheduling and dispatching: A review of models and algorithms. Proc. of the 5th Workshop on Algorithmic Methods and Models for Optimization of Railways, Dagstuhl Research Online Publication Server: Sweden, 2006.

[14] Shaw-Ching Chang, Y.C.C., From timetabling to train regulation - a new train operation model. Information and Software Technology, 47, pp. 575585, 2005.

[15] Walker, C.G., Snowdon, J.N. \& Ryan, D.M., Simultaneous disruption recovery of a train timetable and crew roster in real time. Computers \& Operations Research, 32(8), pp. 149-2094, 2005. 